Institut fur Plasmaphysik Association EUAATOM-KFA

Measurement of the Vertical Magnetic Field in TEXTOR Using Electron Beam Technique
O. Neubauer
F.H. Bohn
A. Chudnovskij
B. Giesen
P. Hüttemann
M. Lochter 




Berichte des Forgchumgizentrums witich: 3032 SSWN 0944-29:5.

Institut fir Plesmanhysik Jul-3032

Associkor EURATOW HEA

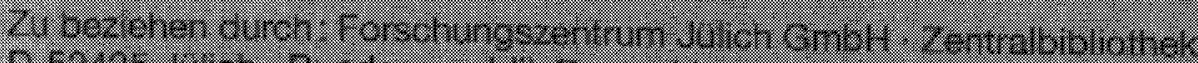

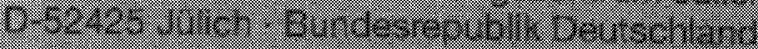

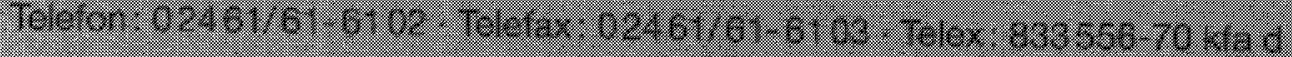




\section{Measurement of the Vertical Magnetic Field in TEXTOR Using Electron Beam Technique}
O. Neubauer
F.H. Bohn
A. Chudnovskij*
B. Giesen
P. Hüttemann
M. Lochter

"Kurchatov Institute, Moscow 
Measurement of the Vertical Magnetic Field in TEXTOR Using Electron Beam Technique

\section{Abstract}

Determination of the vertical stray field in the plasma centre produced by poloidal field coils is essential for the control of breakdown and of the horizontal plasma position in tokamaks. Measurement by magnetic probes does not provide satisfactory results since the toroidal field perpendicular to the poloidal one is $30 \ldots 3000$ times higher and thus induces error signals:

Electron beam technique has been chosen to solve this problem. Therefore an electron gun has been specially designed for TEXTOR and implemented for measurements.

Magnetic field lines "became visible" using the effect of electron movement in magnetic fields and light emission in a gas. Vertical fields were determined from the measured toroidal field and vertical electron beam displacements.

In addition to the measured efficiencies of poloidal field coils, exact values for the field compensation during premagnetization have been defined.

Results have been verified by field calculations and compared with plasma breakdown experiments. 


\section{Contents}

1 Why Magnetic Field Measurements ? .........................................

2 Method of Field Measurement .................................................. 8

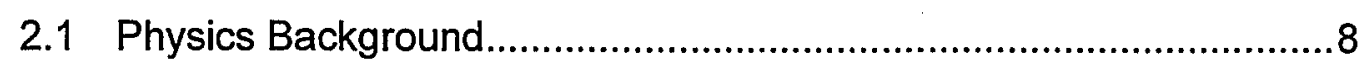

2.2 Technical Description..............................................................9

2.3 Determination of the Field from the Beam Displacement................ 10

2.4 Experimental Parameters...................................................... 11

3 Results of Vertical Field Measurements..................................... 12

3.1 Efficiency of BM Coils - Inductor Coils .......................................13

3.2 Efficiency of BV Coils - Position Control Coils............................... 14

3.3 Efficiency of BF Coils - Shaping Coils .......................................... 15

3.4 Efficiency of KV Coils - Position Control Coils................................16

3.5 Compensation of BM Field ..................................................... 17

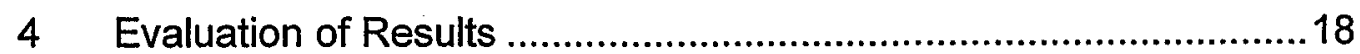

4.1 Comparison with Calculated Efficiencies ......................................18

4.2 Results of Stray Field Compensation during Premagnetization ....... 19

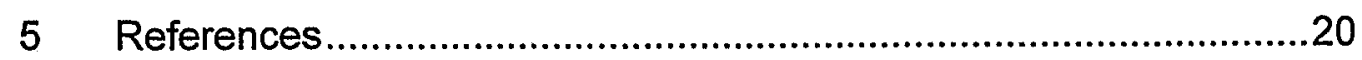


One main operating principle of a tokamak like TEXTOR is the confinement of charged particles in plasma by means of a magnetic field. Electrons and ions in TEXTOR move along magnetic field lines with a toroidal component (up to $2.8 \mathrm{~T}$ ) and a poloidal component (up to some $100 \mathrm{mT})$.

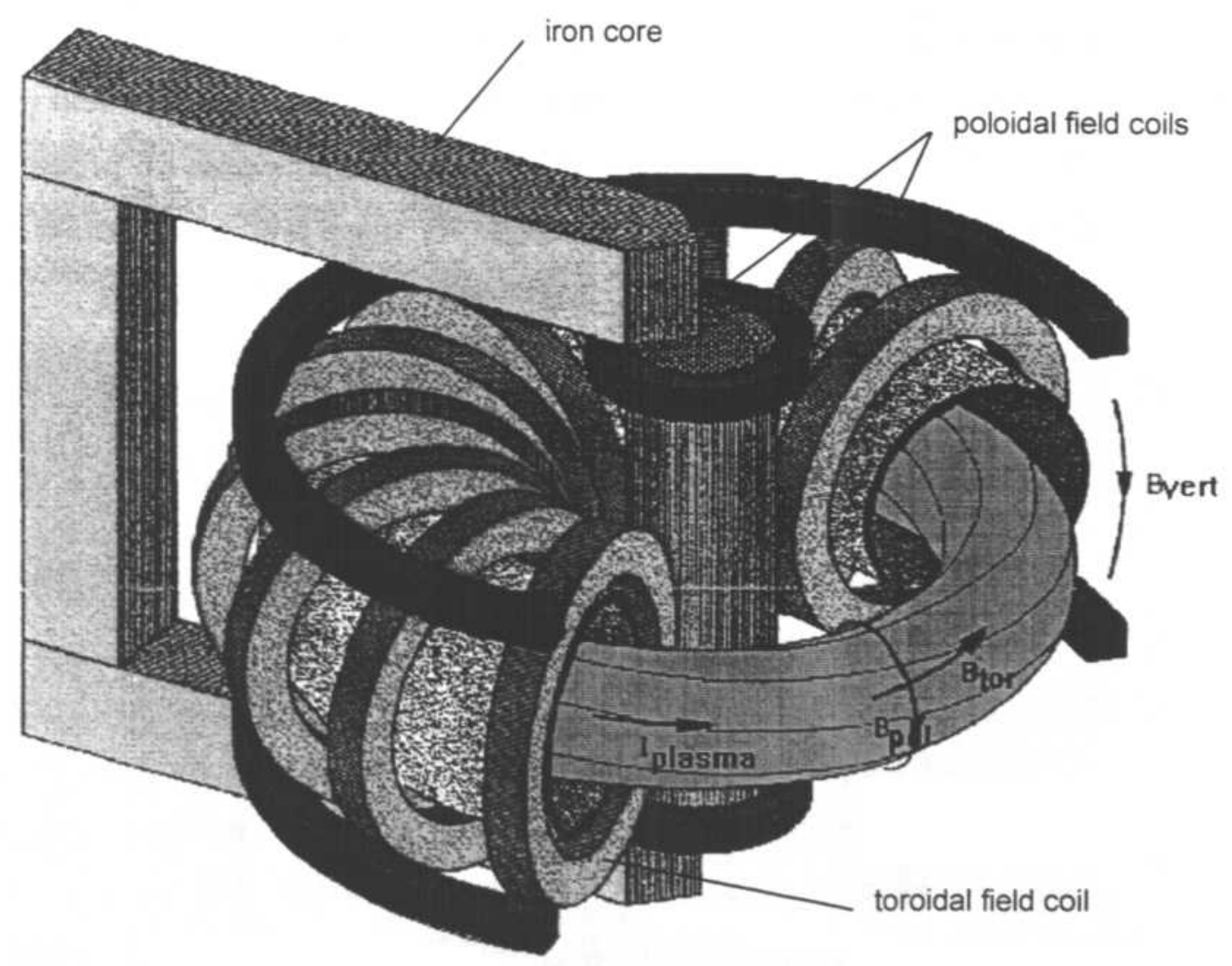

Fig. 1. Tokamak principle.

The latter is produced by a set of poloidal field coils for ohmic heating, shaping and position control of the plasma, all with currents variable in time, and by the plasma itself whereas the main magnetic field (toroidal) is generated by 16 specially arranged toroidal field coils carrying a constant current during the whole plasma discharge.

Regarding the poloidal field, there are two aspects of interest: 
(1) For optimal control of plasma position and shape the poloidal field produced by each coil in the plasma volume during discharge (coil efficiency) has to be determined.

(2) For plasma breakdown the vertical field has to be as low as possible (by compensation) thus particles remain in the plasma volume as long as possible and giving better conditions for ionization.

During plasma operation the TEXTOR diagnostic system already provides some vertical field measurements with magnetic probes outside the vessel. Due to the large ratio between the toroidal and poloidal field (order of $30 \ldots 3000$ ) and the influence of horizontal components on probe coils their precision is limited. For that reason for more precise measurements even in the plasma centre an electron beam method was chosen.

\section{The Method of Field Measurement}

\subsection{Physics Background}

Charged particles with an initial velocity are „captured" by a magnetic field. The velocity component parallel to the field remains unaffected by the field whereas the component perpendicular to the field produces a rotation round the field line caused by the Lorenz force. The radius of the electron's rotation is less than $1 \mathrm{~cm}$ in case of our experiment.

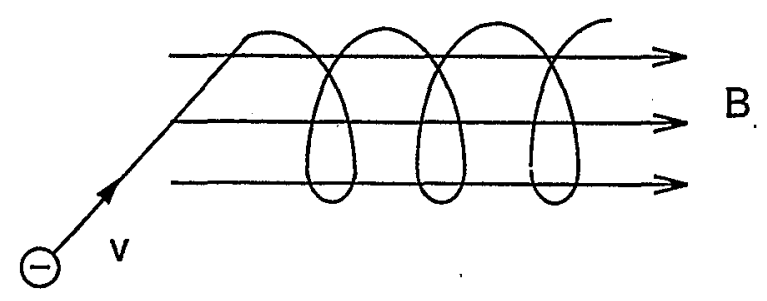

Fig. 2. Movement of electrons in a magnetic field. 
Electrons are brought into the TEXTOR vacuum vessel and accelerated. By making the electron's way visible in an appropriate gas, which is excited for radiation by collisions with electrons, one can directly "observe" magnetic field lines as light beam. In this case the gas atmosphere has to be highly diluted.

\subsection{Technical Description}

The method described above was realised in TEXTOR by an electron gun 11/. A special introduction device which allows arbitrary vertical positioning without disturbing the vacuum was designed. It places an ordinary vacuum diode without a glass tube emitting electrons in the vacuum chamber filled either with argon or hydrogen at about $10^{-3} \mathrm{Torr}$.

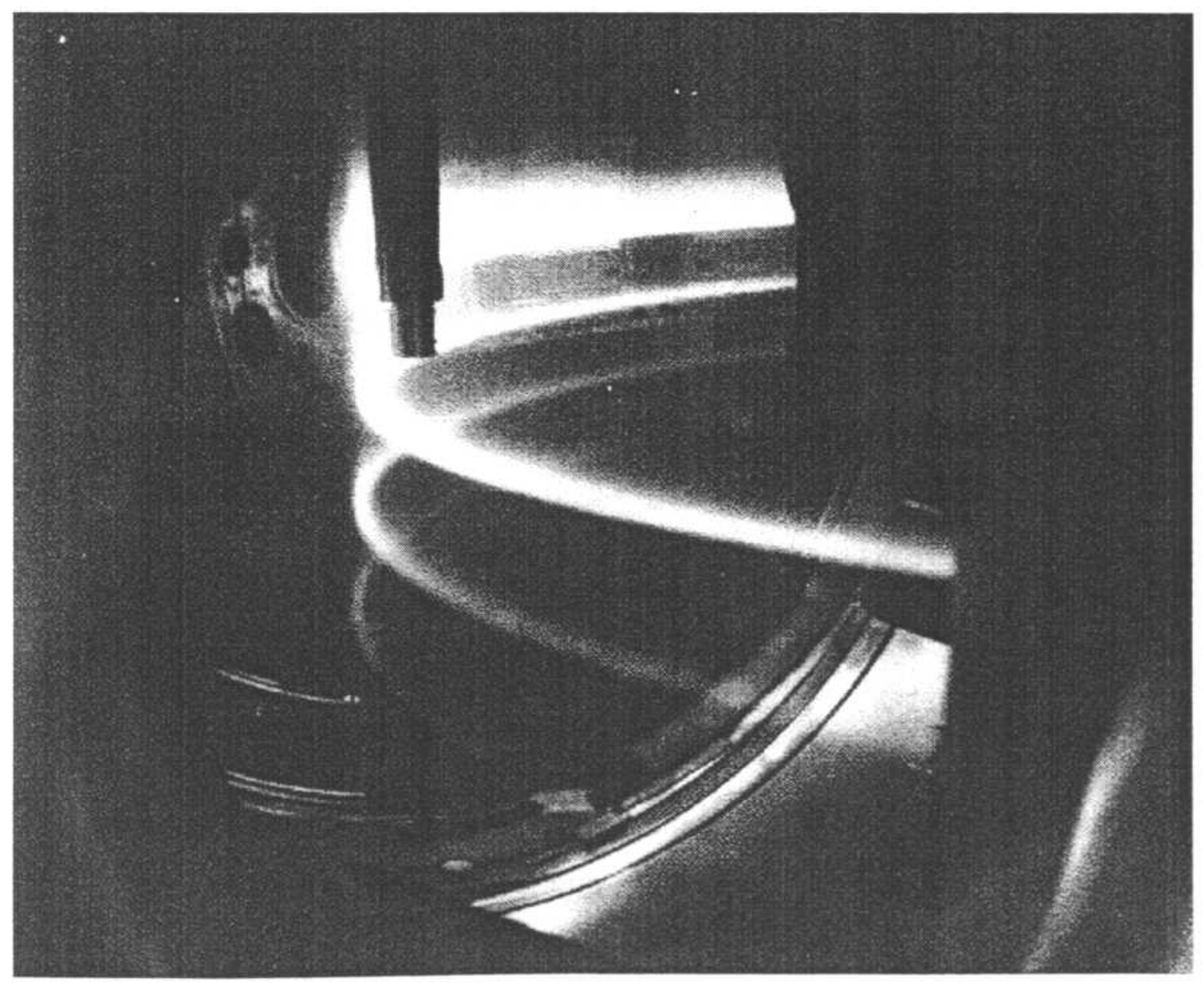

Fig. 3. View into vacuum vessel with electron beam. 
The electrons were accelerated by an anode voltage. A constant toroidal field held the electrons in a circular orbit. Additional small vertical fields produced deflection forces which resulted in helical orbits. The value of the vertical field was determined by the vertical displacement per turn of the electron beam.

2.3 Determination of the Field from the Beam Displacement

The pass of the electron beam can be described by figure 4 ,



Fig. 4. Field components.

where $z$ and $\varphi$ are the vertical and toroidal coordinates, $B_{v e r t}$ and $B_{t o r}$ the vertical and toroidal field densities and $R$ the radial position of the electron gun.

The vertical displacement after $n$ revolutions is

$$
\Delta z=\int_{0}^{n 2 \pi} \frac{B v e r t(\varphi)}{B_{t o r}(\varphi)} R d \varphi
$$

Assuming a constant toroidal field, we find

$$
\Delta z \approx \frac{\overline{B_{\text {vert }}}}{\overline{B_{\text {tor }}}} n 2 \pi R .
$$

The accuracy of determining absolute vertical field values mainly depends on the measurement of displacement and is estimated to be about $10 \%$. The more revolutions of the beam are visible the less the error is. 
However, the method allows very exact measurements of the field compensation. After one revolution at a vertical displacement of $1 \mathrm{~cm}$ and a major radius of $1.75 \mathrm{~m}$ the vertical field is

$$
\overline{B_{\text {vert }}} \approx \frac{\Delta z}{2 \pi R_{0}} \overline{B_{\text {tor }}} \approx 10^{-3} \overline{B_{\text {tor }}}
$$

That means the vertical stray field may be compensated to $1 m T$ at $\overline{B_{t o r}}=1 T$ and at lower toroidal fields even lower.

\subsection{Experimental Parameters}

The parameters of the experiments were adjusted according to $/ 2 /$. The electron gun was placed at the centre of the vacuum vessel.

The emission current was about $5 \mathrm{~mA}$.

Electrons were accelerated by an anode voltage of about $80 \mathrm{~V}$.

During operation with hydrogen the pressure was $1.0510^{-3} \mathrm{mbar}$. For toroidal fields above $20 \mathrm{mT}$ argon had to be used because the beam was no longer visible in hydrogen. The pressure in argon was chosen at $2.710^{-3} \mathrm{mbar}$.

An usual CCD video camera with an image intensifier (sensitivity: $1 \mu \mathrm{Lx}$ ) and an exposure of $20 \mathrm{~ms}$ was installed to observe the electron beam through a window of the TEXTOR vessel. The camera provided safety in observation of the beam and more reliability of displacement data. The beam displacement was determined directly from the monitor. However, digitally processing is also possible by applying image processing methods on the video pictures. 
During electron beam experiments, all poloidal field coils of TEXTOR were fed separately, covering the typical range of operation. In addition a wide range of stray fields produced by currents in the inductor coils was compensated by either the plasma shaping coils or the position control coils to obtain current values for the stray field compensation during the premagnetization phase.

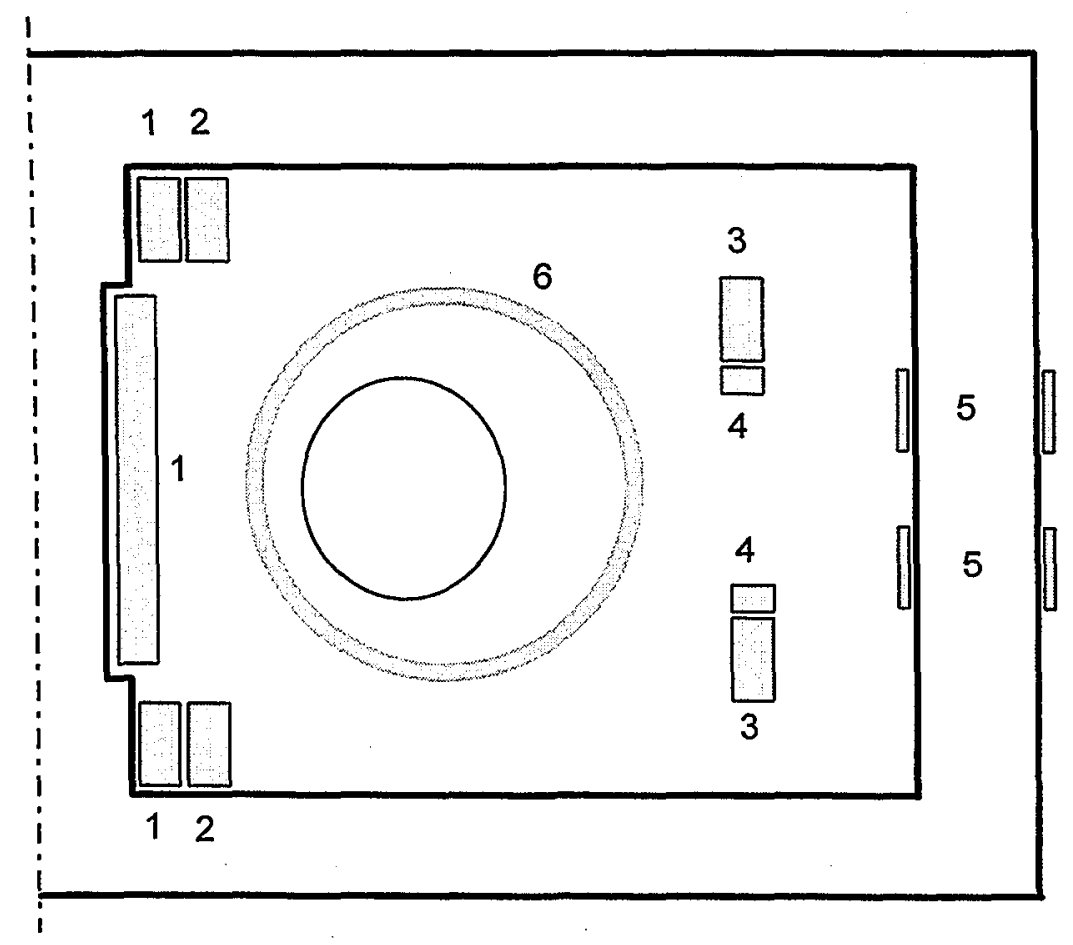

Fig. 5. Arrangement of the TEXTOR coils. Vertical cut through the yoke plane.

1 Inductor coils (BM),

2 Shaping coils (BF),

3 Position control coils (BV),

4 Position control coils (KV) in series with 5 ,

6 Toroidal field coils. 
3.1 Efficiency of BM Coils - Inductor Coils

\begin{tabular}{|c|c|c|c|c|c|c|}
\hline Shot No. & $\mathrm{I}_{\mathrm{BM}}$ & $I_{\text {to }}$ & $B_{\text {tor }}$ & $\Delta \mathrm{z}$ & $B_{\text {vert }}$ at $R_{0}$ & Efficiency \\
\hline & {$[\mathrm{kA}]$} & {$[\mathrm{kA}]$} & {$[\mathrm{mT}$} & {$[\mathrm{m}]$} & {$[\mathrm{mT}$} & {$[\mathrm{mT} / \mathrm{kA}]$} \\
\hline $\mathrm{BM} 3$ & 0.250 & 0.20 & $\overline{7.4}$ & 0.35 & 0.236 & 0.942 \\
\hline BM2 & 0.330 & 0.40 & 14.8 & 0.35 & 0.471 & 1.428 \\
\hline 57400 & 0.392 & 0.70 & 25.9 & 0.29 & 0.671 & 1.713 \\
\hline 57401 & 0.392 & 0.70 & 25.9 & 0.25 & 0.589 & 1.502 \\
\hline BM1 & 0.420 & 0.60 & 22.2 & 0.35 & 0.707 & 1.683 \\
\hline 57398 & 0.624 & 0.70 & 25.9 & 0.46 & 1.084 & 1.736 \\
\hline 57399 & 0.624 & 0. & 25.9 & 0.46 & 1.084 & 1.736 \\
\hline 57533 & 1.900 & 11.50 & 428 & 0.16 & 6.033 & 3.176 \\
\hline 57521 & 3.380 & 11.50 & 428 & 0.22 & 8.564 & 2.534 \\
\hline 57534 & .360 & 11.50 & 428 & 0.23 & 8.953 & 2.053 \\
\hline 57509 & 5.800 & 16.40 & 609 & 0.16 & 8.862 & 1.528 \\
\hline 57510 & 7.760 & 16.40 & 609 & 0.15 & 8.308 & 1.071 \\
\hline 57520 & 10.70 & 16.40 & 609 & 0.17 & 9.416 & 0.880 \\
\hline 57511 & 14.58 & 16.40 & 609 & 0.15 & 8.308 & 0.570 \\
\hline
\end{tabular}

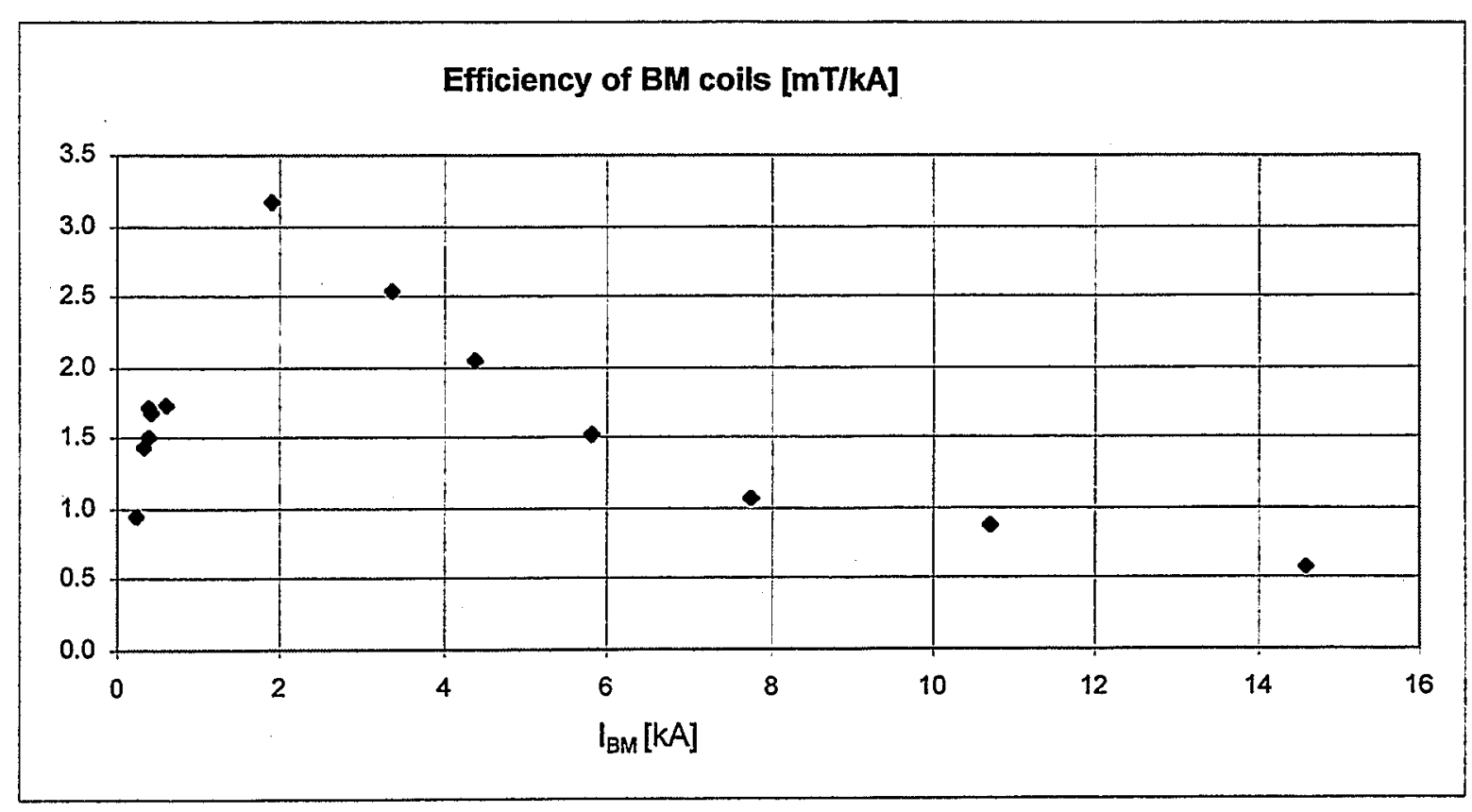

Fig. 6.

Being close to the iron core, the inductor coils show saturation of their efficiency. 
3.2 Efficiency of BV Coils - Position Control Coils

\begin{tabular}{|c|c|c|c|c|c|c|}
\hline Shot No. & $I_{B V}$ & $I_{\text {tor }}$ & $\overline{B_{\text {tor }}}$ & $\Delta z$ & $\mathrm{~B}_{\text {vert }}$ at $\mathrm{R}_{0}$ & Efficiency \\
\hline & {$[\mathrm{kA}]$} & {$[\mathrm{kA}]$} & {$[\mathrm{mT}$} & {$[\mathrm{m]}$} & ImT & {$[\mathrm{mT} / \mathrm{kAl}$} \\
\hline 57406 & 0.029 & 0.7 & 25.9 & 0.20 & 0.471 & 16.25 \\
\hline 57408 & 0.066 & 0.7 & 25.9 & 0.44 & 1.036 & 15.70 \\
\hline 407 & 0.067 & 0.7 & 25.9 & $\overline{0.44}$ & 1.036 & 15.47 \\
\hline 537 & 0.386 & 11.5 & 426 & 0.15 & 5.805 & 15.04 \\
\hline 536 & 0.9 & 1 & 607 & 0.25 & 13.631 & 15.15 \\
\hline 514 & 1. & 4 & 607 & 0.37 & 20.419 & 14.80 \\
\hline 57525 & 1.900 & 35.7 & 1,321 & 0.26 & 31.235 & 16.44 \\
\hline 57526 & 1.900 & 45.1 & 1,669 & 0.22 & 33.388 & 17.57 \\
\hline 57538 & 2.870 & 45.1 & 1,669 & 0.31 & 47.047 & 16.39 \\
\hline
\end{tabular}

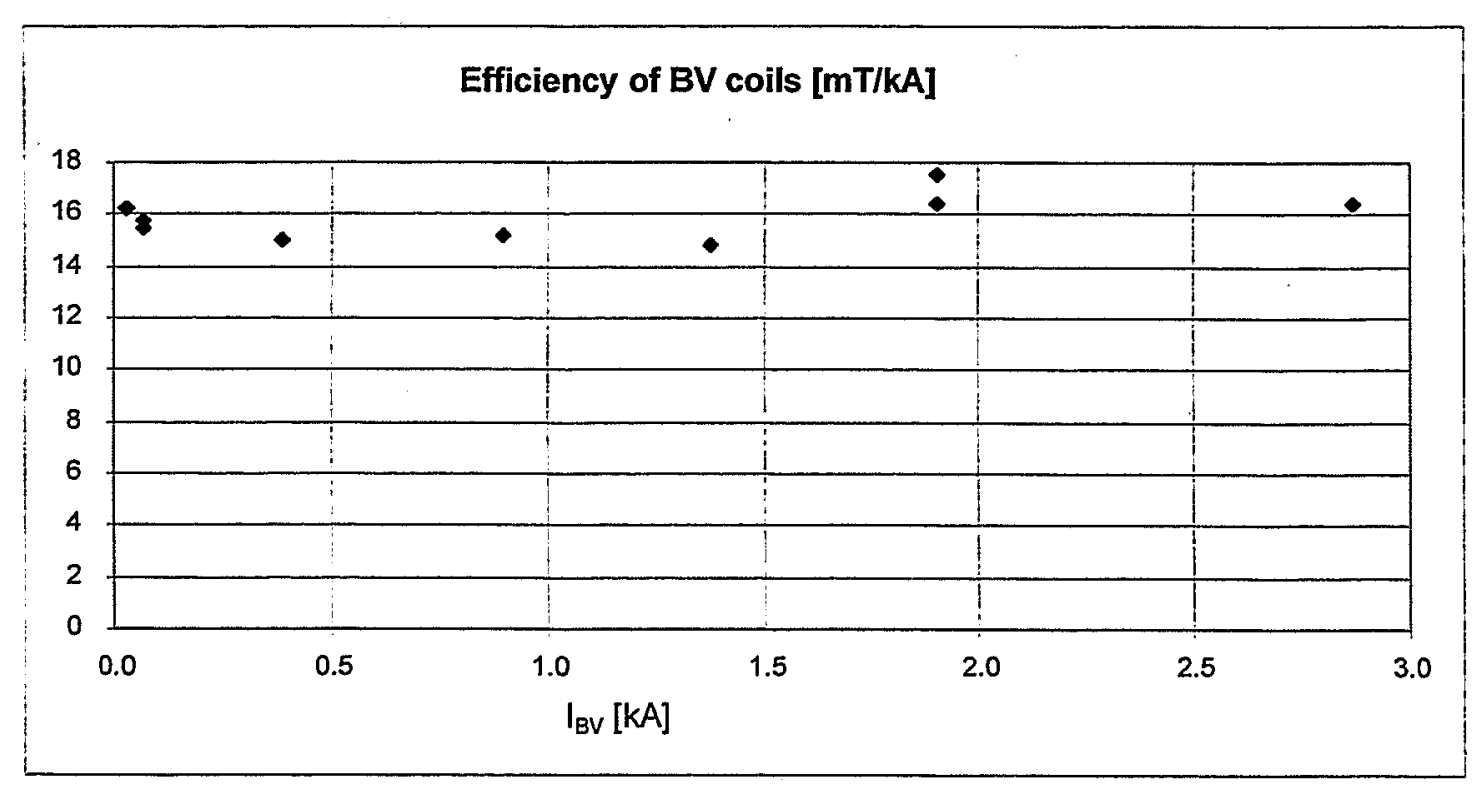

Fig. 7.

Position control coils have a considerable distance to the iron core thus like an air coil showing almost constant efficiency. 
3.3 Efficiency of BF Coils - Shaping Coils

\begin{tabular}{|c|c|c|c|c|c|c|}
\hline Shot No. & $I_{B E}$ & $\overline{I_{\text {tor }}}$ & $B_{\text {tor }}$ & $\Delta z$ & $\mathrm{~B}_{\text {vert }}$ at $\mathrm{R}_{0}$ & Efficiency \\
\hline & {$[\mathrm{kA}]$} & {$[\mathrm{kA}]$} & {$[\mathrm{mT}]$} & [m] & {$[\mathrm{mT}]$} & {$[\mathrm{mT} / \mathrm{kA}]$} \\
\hline BF3 & 0.14 & 0.2 & 7.4 & 0.350 & 0.236 & 1.68 \\
\hline $\mathrm{BF} 2$ & 0.23 & 0.4 & 14.8 & 0.350 & 0.471 & 2.05 \\
\hline BF1 & 0.32 & 0.6 & 22.2 & 0.350 & 0.707 & 2.21 \\
\hline 57527 & 1.97 & 11.5 & 425.5 & 0.170 & 6.579 & 3.34 \\
\hline 57518 & 4.76 & 16.4 & 606.8 & 0.230 & 12.693 & 2.67 \\
\hline 57517 & 9.70 & 16.4 & 606.8 & 0.345 & 19.040 & 1.96 \\
\hline 57515 & 14.60 & 16.4 & 606.8 & 0.440 & 24.282 & 1.66 \\
\hline
\end{tabular}

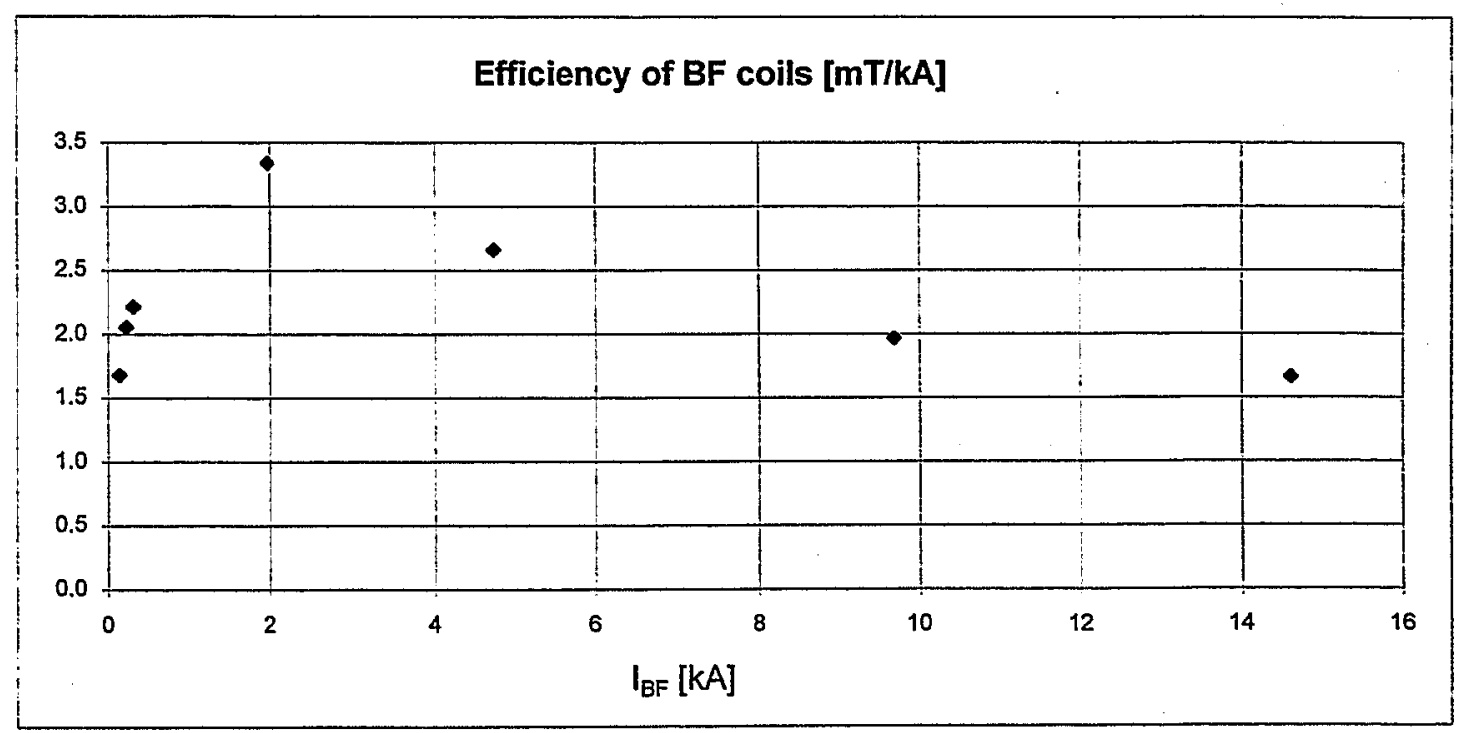

Fig. 8.

Shaping coils close to inductor coils have similar characteristics (apart from the total value depending on the number of turns). 
3.4 Efficiency of KV Coils - Position Control Coils

\begin{tabular}{|l|l|r|r|r|r|r|}
\hline Shot No. & $I_{\mathrm{KV}}$ & \multicolumn{1}{l|}{$\mathrm{I}_{\text {tor }}$} & \multicolumn{1}{l|}{$\mathrm{B}_{\text {tor }}$} & \multicolumn{1}{l|}{$\Delta \mathrm{z}$} & \multicolumn{1}{l|}{$\mathrm{B}_{\text {vert }}$ at $\mathrm{R}_{0}$} & Efficiency \\
\hline & {$[\mathrm{kA}]$} & \multicolumn{1}{l|}{$[\mathrm{kA}]$} & \multicolumn{1}{l}{$[\mathrm{mT}]$} & \multicolumn{1}{l|}{$[\mathrm{m}]$} & \multicolumn{1}{l}{$[\mathrm{mT}]$} & {$[\mathrm{mT} / \mathrm{kA}]$} \\
\hline 57413 & 0.313 & 0.70 & 25.9 & 0.180 & 0.424 & 1.355 \\
\hline 57414 & 0.609 & 0.70 & 25.9 & 0.300 & 0.707 & 1.160 \\
\hline 57415 & 0.917 & 0.70 & 25.9 & 0.400 & 0.942 & 1.028 \\
\hline 57416 & 1.150 & 0.70 & 25.9 & 0.440 & 1.036 & 0.901 \\
\hline 57531 & 1.190 & 6.77 & 250.5 & 0.050 & 1.139 & 0.957 \\
\hline 57544 & 2.160 & 3.78 & 139.9 & 0.132 & 1.679 & 0.777 \\
\hline 57543 & 2.930 & 6.77 & 250.5 & 0.111 & 2.529 & 0.863 \\
\hline 57532 & 2.980 & 6.77 & 250.5 & 0.126 & 2.870 & 0.963 \\
\hline
\end{tabular}



Fig. 9

The KV position control coils act differently to the BV coils since their effect on the iron core and additionally a fraction of the stray field is compensated by the yoke coils (see fig. 5 , position 5 ) connected in series with KV coils. 


\subsection{Compensation of BM Field}

$\mathrm{B}_{\text {tor }}=26 \mathrm{mT}, \Delta \mathrm{z}<3 \mathrm{~cm}, \mathrm{~B}_{\text {vert }}<0.1 \mathrm{mT}$

\begin{tabular}{|l|r|r|}
\hline Shot No. & $I_{\mathrm{BM}}$ & \multicolumn{1}{l|}{$\mathrm{I}_{\mathrm{BV}}$} \\
\hline & {$[\mathrm{kA}]$} & {$[\mathrm{kA}]$} \\
\hline 57433 & 1.07 & 0.140 \\
\hline 57434 & 1.07 & 0.150 \\
\hline 57438 & 4.95 & 0.450 \\
\hline 57449 & 9.90 & 0.490 \\
\hline 57455 & 14.67 & 0.494 \\
\hline 57459 & 19.45 & 0.415 \\
\hline 57462 & 23.97 & 0.333 \\
\hline
\end{tabular}

\begin{tabular}{|l|r|r|}
\hline Shot No. & \multicolumn{1}{|l|}{$\mathrm{I}_{\mathrm{BM}}$} & \multicolumn{1}{l|}{$\mathrm{I}_{\mathrm{BF}}$} \\
\hline & {$[\mathrm{kA}]$} & {$[\mathrm{kA}]$} \\
\hline 57468 & 14.70 & 5.20 \\
\hline 57469 & 14.70 & 5.38 \\
\hline 57471 & 13.68 & 5.40 \\
\hline 57473 & 9.80 & 5.31 \\
\hline 57475 & 4.94 & 4.01 \\
\hline 57479 & 19.06 & 5.13 \\
\hline 57480 & 23.96 & 3.87 \\
\hline
\end{tabular}



Fig. 10

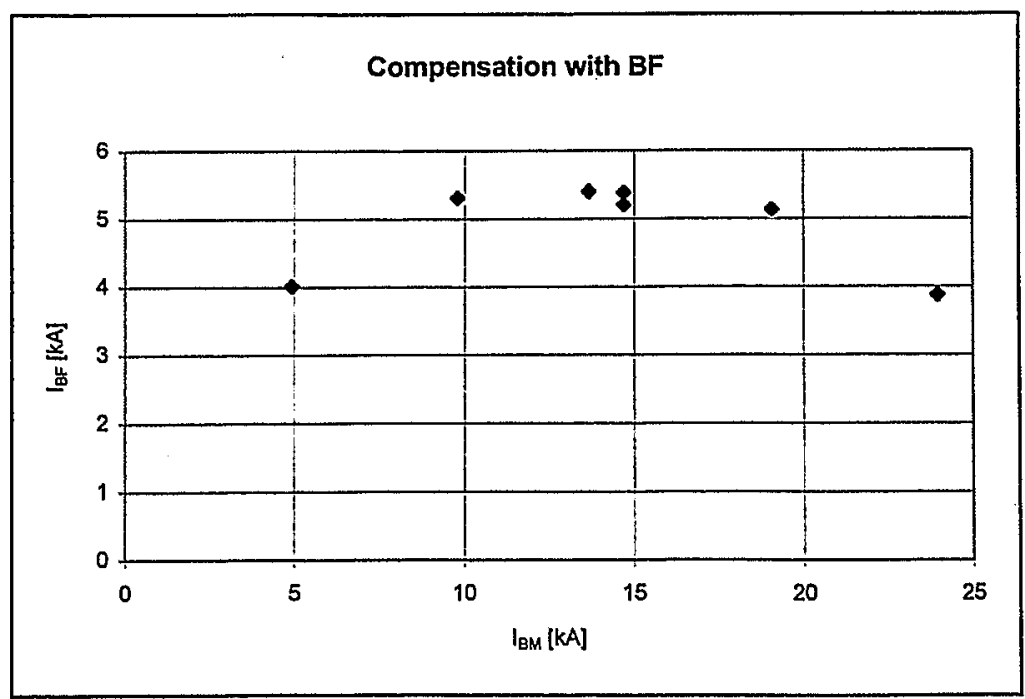

Fig. 11.

The efficiencies of poloidal field coils strongly depend on the saturation of the iron core. Thus the results of stray field compensation are not a simple superposition of single efficiencies 
4 Evaluation of Results

\subsection{Comparison with Calculated Efficiencies}

Due to the principle to measure magnetic fields by the electron beam method, the results are an excellent verification of calculations. Calculations are necessary because electron beam measurements do not work during tokamak operation. Calculations provide information about spatial distribution of stray fields as well.

In the TEXTOR case several 2 dimensional and 3 dimensional models were developed to calculate magnetic fields $/ 3 /, 14 /$.

In $/ 3 /$ a 3 dimensional model is described. Taking into account the symmetry of TEXTOR, only half of the height and $30^{\circ}$ of the circumference had to be modelled. Among others this model is used to calculate the stray field in the plasma region. As small changes or inaccuracies in the model have considerable influence even on the calculated field outside the iron core, designing an adequate model requires experience and verification by experiments.

In order to check the results of the model described above the results of the electron beam measurements provided an appropriate basis. A comparison between measurements and calculations proved the suitability of the 3 dimensional model. 
4.2 Results of Stray Field Compensation during Premagnetization

During plasma operation with premagnetization of the iron core leading to disturbing stray fields, the results of electron beam measurements were helpful to find improved conditions for plasma breakdown. The values given in figure 10 and figure 11 provide the necessary compensation currents either in position control coil or in shaping coil for the whole range of premagnetization currents in the inductor coil.

The accuracy of the electron beam measurements was confirmed by comparison of the results with one set of operating conditions found in experiments at $I_{B M}=14 \mathrm{kA}$ and $I_{B F}=5.4 \mathrm{kA}$. In addition, the results of magnetic probes for stray field measurements confirmed the field compensation under the conditions described above 
/1/ Kurchatov Institute

System for Measurement of Stray Magnetic Fields for TEXTOR Moscow 1993

I2I Vertiporokh, A., Kurchatov Institute

Program of Magnetic Field Measurements by the Electron Beam Technique

Moscow 1994

/3/ Müller, G., Sieling, U.

Vergleich verschiedener Magnetfeldberechnungen von 2D- und 3D-TEXTOR-Modellen

Internal Report: IB-KFA-ZEL-501993

14l Dnestrovskij, Yu. N., Lysenko, S.E., Tsaun, S.V., Vertiporokh, A.N.

Kurchatov Institute

Breakdown in TEXTOR

Moscow 1992 


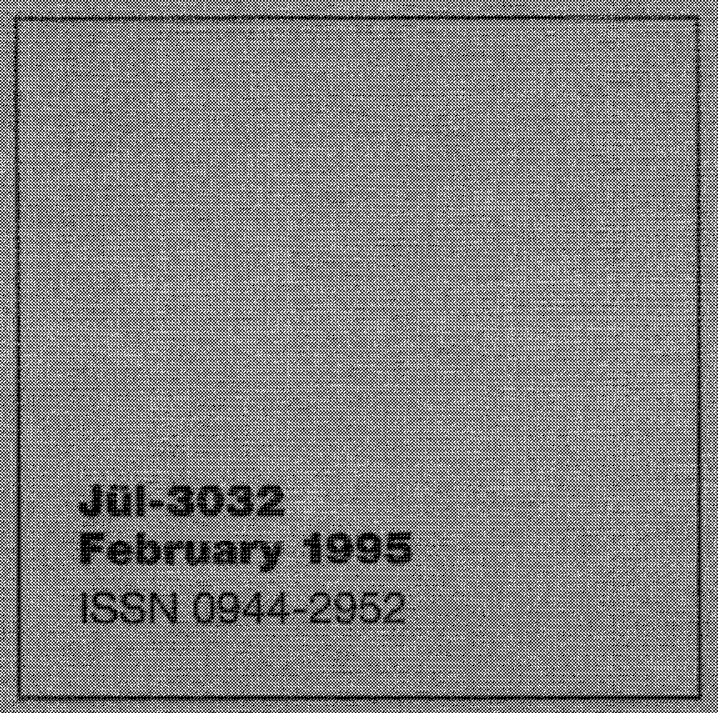

